\title{
The GhREV transcription factor regulate the development of shoot apical meristem in cotton (Gossypium hirsutum)
}

\author{
Doudou YANG ${ }^{1}$, AN Jing ${ }^{1}$, LI Fangjun ${ }^{1}$, ENEJI A. Egrinya ${ }^{2}$, TIAN Xiaoli ${ }^{1 *}$ and LI Zhaohu ${ }^{1}$
}

\begin{abstract}
Background: Manual topping is a routine agronomic practice for balancing the vegetative and reproductive growth of cotton (Gossypium hirsutum) in China, but its cost-effectiveness has decreased over time. Therefore, there is an urgent need to replace manual topping with new approaches, such as biological topping. In this study, we examined the function of GhREV transcription factors (a class III homeodomain-leucine zipper family, HD-ZIP III) in regulating the development of shoot apical meristem (SAM) in cotton with the purpose of providing candidate genes for biological topping of cotton in the future.

Results: We cloned four orthologous genes of AtREV in cotton, namely GhREV1, GhREV2, GhREV3, and GhREV4. All the GhREVs expressed in roots, stem, leaves, and SAM. Compared with GhREV1 and GhREV3, the expression level of GhREV2 and GhREV4 was higher in the SAM. However, only GhREV2 had transcriptional activity. GhREV2 is localized in the nucleus; and silencing it via virus-induced gene silencing (VIGS) produced an abnormal SAM. Two key genes, GhWUSA10 and GhSTM, which involved in regulating the development of plant SAM, showed about 50\% reduction in their transcripts in VIGS-GhREV2 plants.
\end{abstract}

Conclusion: GhREV2 positively regulates the development of cotton SAM by regulating GhWUSA10 and GhSTM potentially.

Keywords: Cotton, Revoluta (REV), Shoot apical meristem (SAM), Virus induced gene silencing (VIGS)

\section{Background}

All aerial organs (leaves, stems, flowers, and germline) of plants are derived from the shoot apical meristem (SAM), which is the basis of aboveground biomass sources for crops. The primordia of an organ arises from the periphery of the SAM and develops into leaves at the vegetative growth stage, or flowers at the reproductive growth stage (Pautler et al. 2013). In dicotyledonous angiosperms, the SAM can be divided into three zones, the central zone $(\mathrm{CZ})$, the organizing center $(\mathrm{OC})$ and the peripheral zone (PZ). The central zone contains three layers (L1-L3) of pluripotent stem cells. Directly underneath the $\mathrm{CZ}$ lies the $\mathrm{OC}$, which is a zonation with signals regulating stem cell maintenance. The daughter cells from the $\mathrm{CZ}$ are laterally displaced into the $\mathrm{PZ}$,

\footnotetext{
*Correspondence: tianxl@cau.edu.cn

'Department of Crop Physiology and Cultivation, College of Agronomy and Biotechnology, China Agricultural University, Beijing 100193, China Full list of author information is available at the end of the article
}

where they proliferate and ultimately differentiate during organogenesis (Bäurle and Laux 2003; Soyars et al. 2016).

The class III homeodomain-leucine zipper (HD-ZIP III) family of transcription factors (TFs) is unique to the plant kingdom; it plays important roles in regulating embryo patterning, meristem formation, organ polarity, vascular development, and meristem function (Mcconnell et al. 2001; Du and Wang 2015; Bustamante et al. 2016; Shi et al. 2016). The HD-ZIP III family of Arabidopsis consists of five members, including REVOLUTA (REV), PHABULOSA (PHB), PHAVOLUTA (PHV), CORONA (CNA) and $A T H B 8$ (Baima et al. 1995; Green et al. 2005). All of these HD-ZIP III proteins possess the HDZIP domain containing a homeodomain (a leucine zipper domain acting on DNA binding and protein dimerization), a steroidogenic acute regulatory protein lipid transfer domain (START), and a MEKHLA domain (Ponting and Aravind 1999; Mukherjee and Bürglin 
2006). Studies with loss-of-function alleles of the HDZIP III family members reveal that loss of $R E V$ gene can lead to apparent defects in apical and axillary meristem development, such as the lack of axillary meristematic tissue, reduced branches, and underdeveloped or even sterile flower structure (Talbert et al. 1995; Otsuga et al. 2001). The rev/phb/phv triple mutant shows enhanced defective phenotype, indicating the functional redundancy of REV, PHB and PHV in regulating SAM formation (Emery et al. 2003). ATHB8 and CAN antagonize $R E V$ in certain tissues, but overlap with $R E V$ in other tissues (Prigge et al. 2005).

Cotton (Gossypium hirsutum) is an important economic crop with an indeterminate growth habit. In order to help balance its vegetative and reproductive growth, manual topping (removal of growth tips) of the main stem is often performed during cotton production in China. However, due to the decreasing labor force and higher labor cost in recent decades, there is a pressing need to develop more efficient techniques, such as biological topping, to replace manual topping. In this study, we cloned and identified four homologs of AtREV genes in cotton (GhREV1, GhREV2, GhREV3, and GhREV4), and found that GhREV2 is a key regulator of the development of SAM. The results shed light on developing biological measures to control growth of the main stem of cotton.

\section{Materials and methods}

\section{Plant materials and growth conditions}

Gossypium hirsutum cv CCRI 41 and Xinshi 17 were used in this study to perform the Agrobacterium-mediated virus-induced gene silencing (VIGS) and quantitative real-time polymerase chain reaction (qRT-PCR) assays. Seeds were germinated in sand and transfered into a pot with $5 \mathrm{~L}$ Hoagland's solution (12 seedlings per pot) after 4 days. The experiment was carried out in a greenhouse at $24 \pm 2{ }^{\circ} \mathrm{C}$ (day) $/ 20 \pm 2{ }^{\circ} \mathrm{C}$ (night), $60 \%$ relative humidity, and $400 \mu \mathrm{mol} \cdot \mathrm{m}^{-2} \cdot \mathrm{s}^{-1}$ light with a $14 \mathrm{~h}$ (light) $/ 10 \mathrm{~h}$ (dark) photoperiod. The nutrient solutions were changed every 4 days. Arabidopsis seedlings were grown in a chamber with $22^{\circ} \mathrm{C}, 60 \%$ relative humidity, and $80 \mu \mathrm{mol} \cdot \mathrm{cm}^{-2} \cdot \mathrm{s}^{-1}$ light with a $14 \mathrm{~h}$ (light) $/ 10 \mathrm{~h}$ (dark) photoperiod for protoplast transient assays.

\section{Protein phylogenetic tree and sequence analysis}

The Basic local alignment search tool (BLAST) in CottonGen (http://www.cottongen.org) was used to search the HD-ZIP III homologs in cotton, the corresponding amino acid sequence was downloaded. The phylogenetic tree of HD-ZIP III homologs in cotton and Arabidopsis was built using the neighbor-joining method in MEGA5. Sequence comparative analysis was aligned using multiple sequence alignment (http://multalin.toulouse.inra.fr/multalin/).

\section{Extraction of RNA and qRT-PCR}

Cotton seedling samples were collected for tissuespecific expression of GhREVs at the sixth leaf stage. Shoot apex samples of VIGS-ed cotton were collected after VIGS-GhCLA1 plants showing complete bleaching of the first and second true leaves. The samples were immediately frozen in liquid nitrogen and stored at $-80^{\circ} \mathrm{C}$. Total RNA was isolated from the samples using a Rapid Extraction Kit for plant RNA (Aidlab N09, Beijing, China), then reversely transcribed into cDNA. The expression of GhREVs, GhWUS10A, and GhSTM in the plants was detected by qRT-PCR. Primers used are listed in Additional file 1: Table S1.

\section{Transcriptional activity assay}

The effector and reporter constructs were used to detect transcriptional activity of GhREVs. The reporter includes four copies of GAL4 upstream activation sequence (UAS), a minimal $35 \mathrm{~S}$ promoter (TATA box included), and a luciferase reporter gene. The effectors contained the GAL4 DNA-binding domain with AtDB5 (negative control), or with AtWRKY29 (positive control) or individual GhREVs under the control of the $35 \mathrm{~S}$ promoter. GhREV1, GhREV2, GhREV3, and GhREV4 were cloned into GAL4 vector via restriction enzyme cloning using $\mathrm{NcoI}$ and StuI, respectively. UBQ10-GUS was added as an internal control for transfection efficiency. The activity of luciferase reporter was detected by an enzyme standard instrument (Power Wave XS2, BioTek, America) after $12 \mathrm{~h}$ incubation.

\section{Subcellular localization}

The subcellular localization of GhREV2 protein was performed in Arabidopsis protoplasts. Full length cDNA of GhREV2 was cloned via restriction enzymes using SmaI and $K p n I$ into the pSuper1300 vector to generate $\mathrm{pSu}$ per::GhREV2-GFP. The fused constructs were transformed or co-transformed into protoplasts for $12 \mathrm{~h}$. The fluorescence was examined by a confocal microscopy (ZEISS710, Carl Zeiss, Germany).

\section{Agrobacterium-mediated VIGS}

A 330 bp cDNA fragment of GhREV2 was amplified and cloned into pYL156 (pTRV:RNA2) vector. The primers are listed in Additional file 1: Table S1. Plasmids of binary TRV vectors pTRV:RNA1 and pTRV:RNA2 (Ctrl, GhCLA1, GhREV2) were transformed into Agrobacterium tumefactions strain GV3101 by electroporation. Agrobacterium strains were cultured for VIGS assays as previously described ( $\mathrm{Mu}$ et al. 2019). The mixtures of Agrobacterium strains were infiltrated into two fully expanded cotyledons using a needle-less syringe (Li et al. 2015). 


\section{Data analysis}

Data was pooled across independent repeats. The statistical analyses was performed using one-way analysis of variance (ANOVA), and treatment means were compared using Duncan's multiple range test at $P<0.05$.

\section{Results}

\section{Phylogenetic analysis of HD-ZIP III family}

The HD-ZIP III family in Arabidopsis has been well characterized (Byrne 2006; Youn-Sung et al. 2008; Turchi et al. 2015). The full amino acid sequence of the HD-ZIP III family members in Arabidopsis was used as the query for a BLAST analysis against the G. hirsutum National Biological Information (NBI) protein database (https://www.cottongen.org/blast/). Phylogenetic analysis showed 18 putative HD-ZIP III members in cotton (Fig. 1a), including eight AtREV paralogs, four genes located in the D subgenome and other four genes in the A subgenome. They were named as GhREV1A and GhREV1D (Gh_A05G0892 and Gh_D05G0975), GhREV2A and GhREV2D (Gh_ A03G0276 and Gh_D03G1290), GhREV3A and GhREV3D (Gh_A08G1765 and Gh_D08G2109), and GhREV4A and GhREV4D (Gh_A13G2011 and Gh_ D13G2409) (Fig. 1b), respectively. These GhREVs share 95\%-99\% similarity in their amino acid sequence. In addition, each GhREV shares more than $82 \%$ amino acid identity and $90 \%$ cDNA sequence similarity with Arabidopsis REV. Because of the high similarity of A subgenome and D subgenome (Fig. 1b), GhREVsA and GhREVsD cannot be distinguished by RT-PCR. Thus, we named GhREVsA/D as GhREV1, GhREV2, GhREV3 and GhREV4, respectively, in the following work.

\section{Spatial and temporal expression pattern of GhREVs genes}

The expression levels of genes tend to be correlated with their biological functions. Total RNA of roots, stem, leaf, and shoot apex were extracted from cotton seedlings at the cotyledon stage and at the $2^{\text {nd }}, 4^{\text {th }}, 6^{\text {th }}$, and $8^{\text {th }}$ leaf stages. Quantitative real-time PCR (qRT-PCR) was performed to determine the temporal and spatial transcription expression patterns of GhREVs. The results showed that GhREV genes were expressed in all of the tested tissues, higher in the stem and SAM (Fig. 2). GhREV2 and GhREV4 showed higher expression levels than GhREV1 and GhREV3 in roots, leaves, and the SAM, while the stem possessed more GhREV3 transcript in addition to GhREV2 and GhREV4 (Fig. 2). Considering the temporal expressing pattern of GhREVs in roots (Fig. 2a), leaves (Fig. 2c), and shoot apex
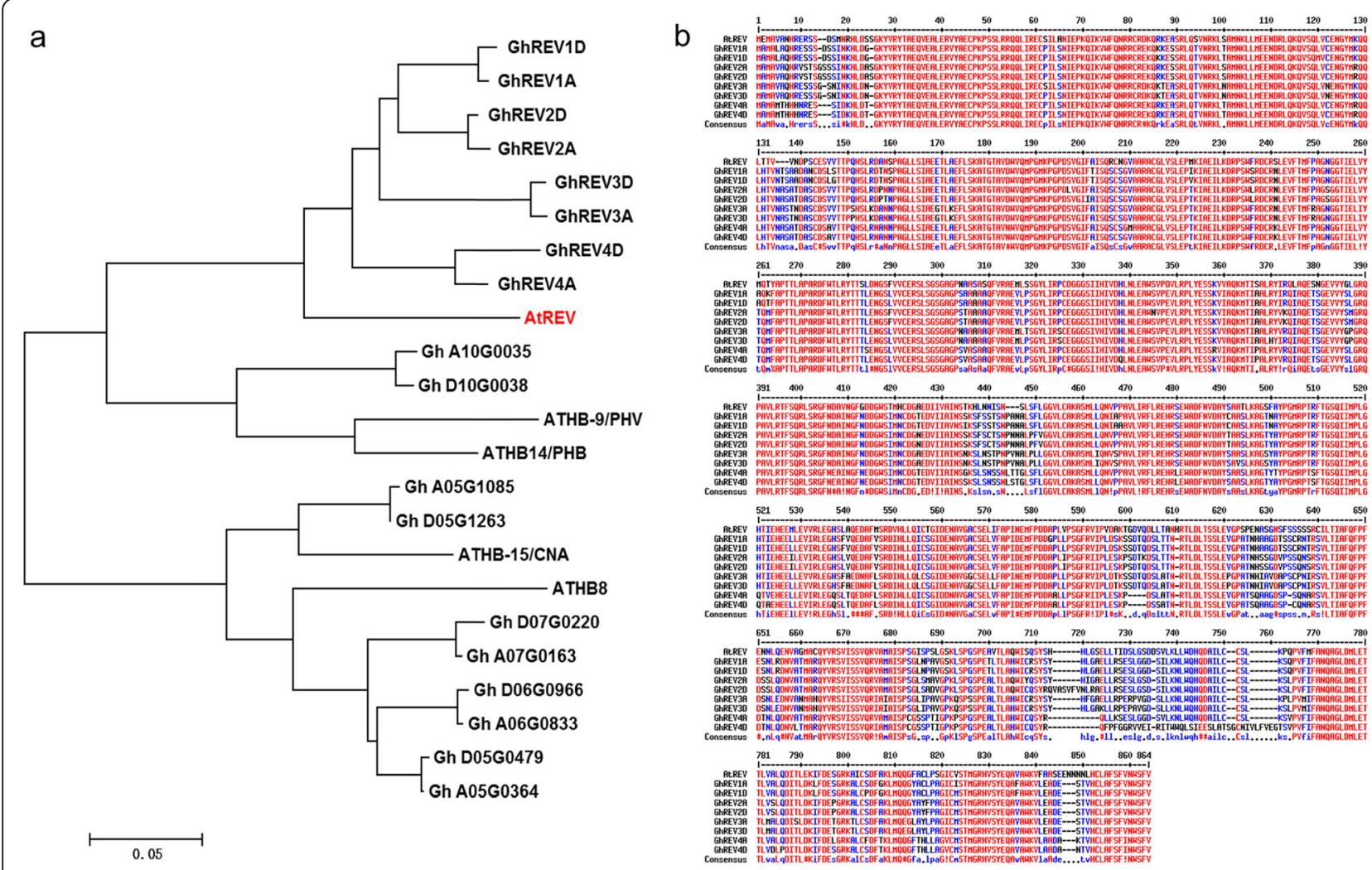

Fig. 1 Sequence analysis of Gossypium hirsutum REVOLUTA (GhREV). (a) Phylogenetic tree of HD-ZIP III family in both cotton and Arabidopsis. The tree was drawn to scale with branch lengths in the same unit. (b) The amino acid sequence alignment of REV in cotton and Arabidopsis 


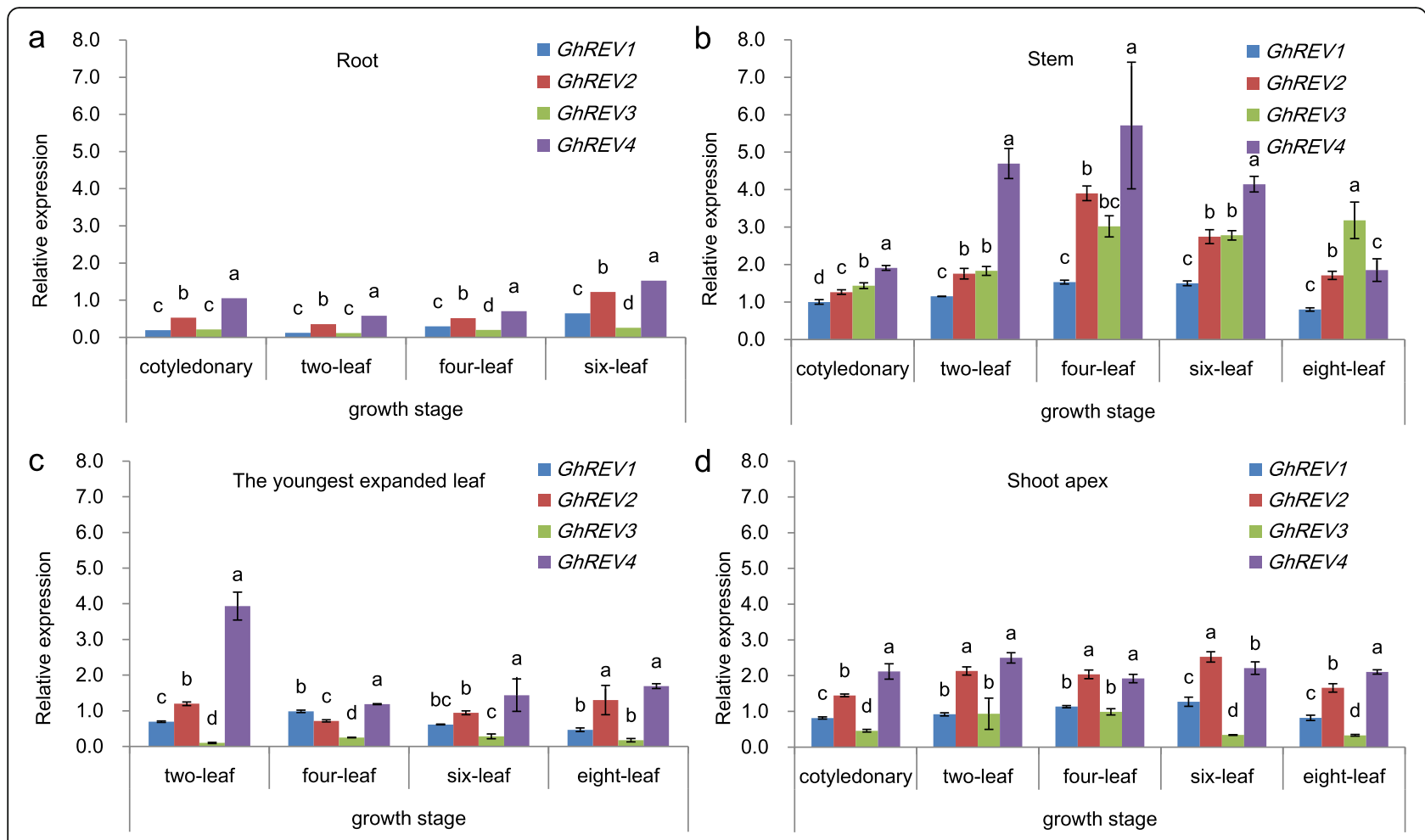

Fig. 2 The expression levels of GhREVs family members in root (a), stem (b), the youngest expanded leaf (c) and shoot apex (d) at cotyledonary, the $2^{\text {nd }}, 4^{\text {th }}, 6^{\text {th }}$, and $8^{\text {th }}$ leaf stages. The expression of GhREV1 in stem was regarded as ' 1 '. GhActing was used as the internal control. There were no data of roots at the $8^{\text {th }}$ leaf stage due to the failure of RNA extraction

(Fig. 2d), there were no obvious and explicit differences from the cotyledon stage to the $6^{\text {th }}$ or $8^{\text {th }}$ leaf stage. For the stem, we observed that the expression level of GhREV2 and GhREV4 peaked at the $4^{\text {th }}$ leaf stage, while GhREV3 peaked at the $8^{\text {th }}$ leaf stage (Fig. 2b).
GhREV2 and GhREV3 act as transcriptional activators To determine whether GhREVs confer transcriptional activity, we carried out an Arabidopsis protoplast-based transactivation assay (Fig. 3a). Compared with the negative control, GhREV2 and GhREV3 significantly

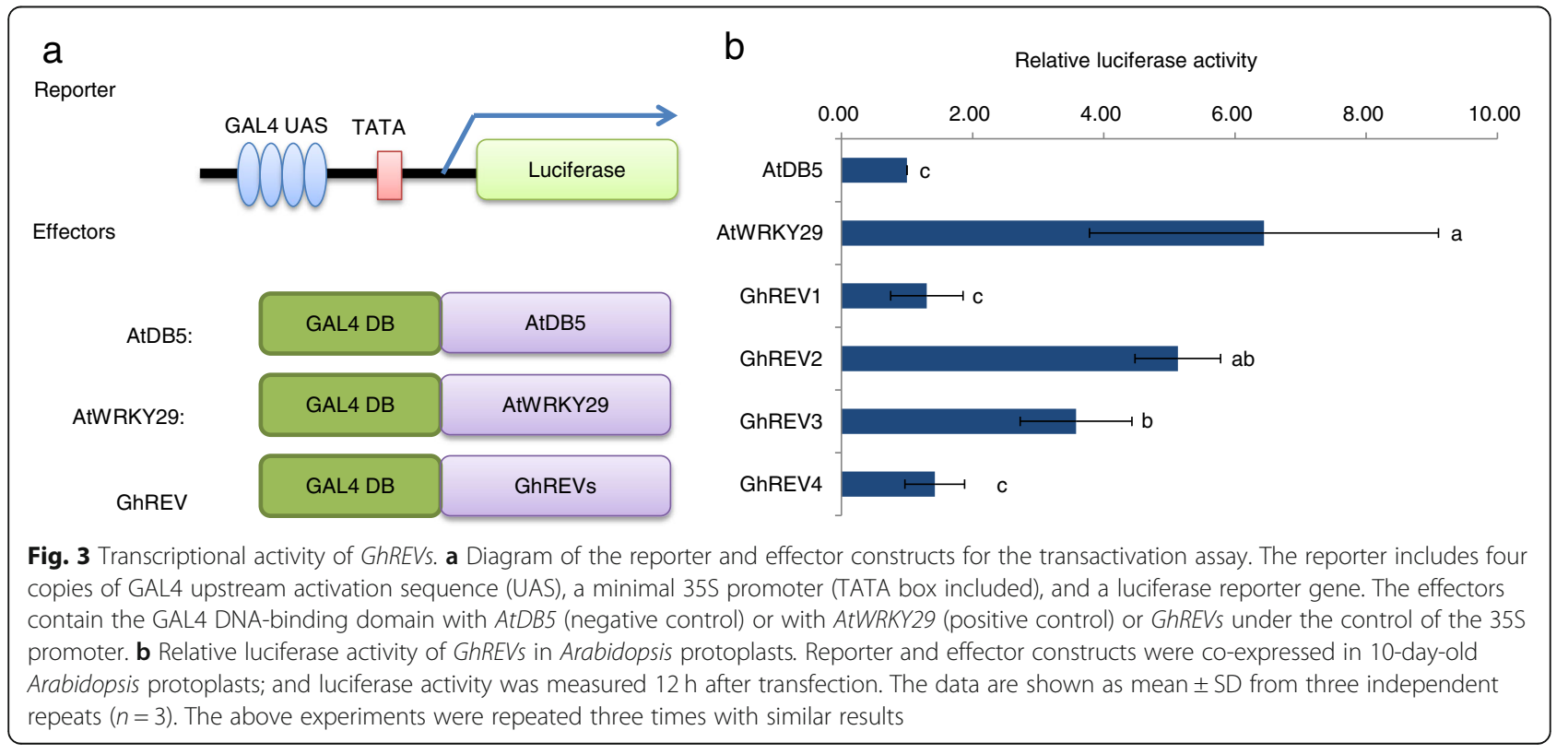




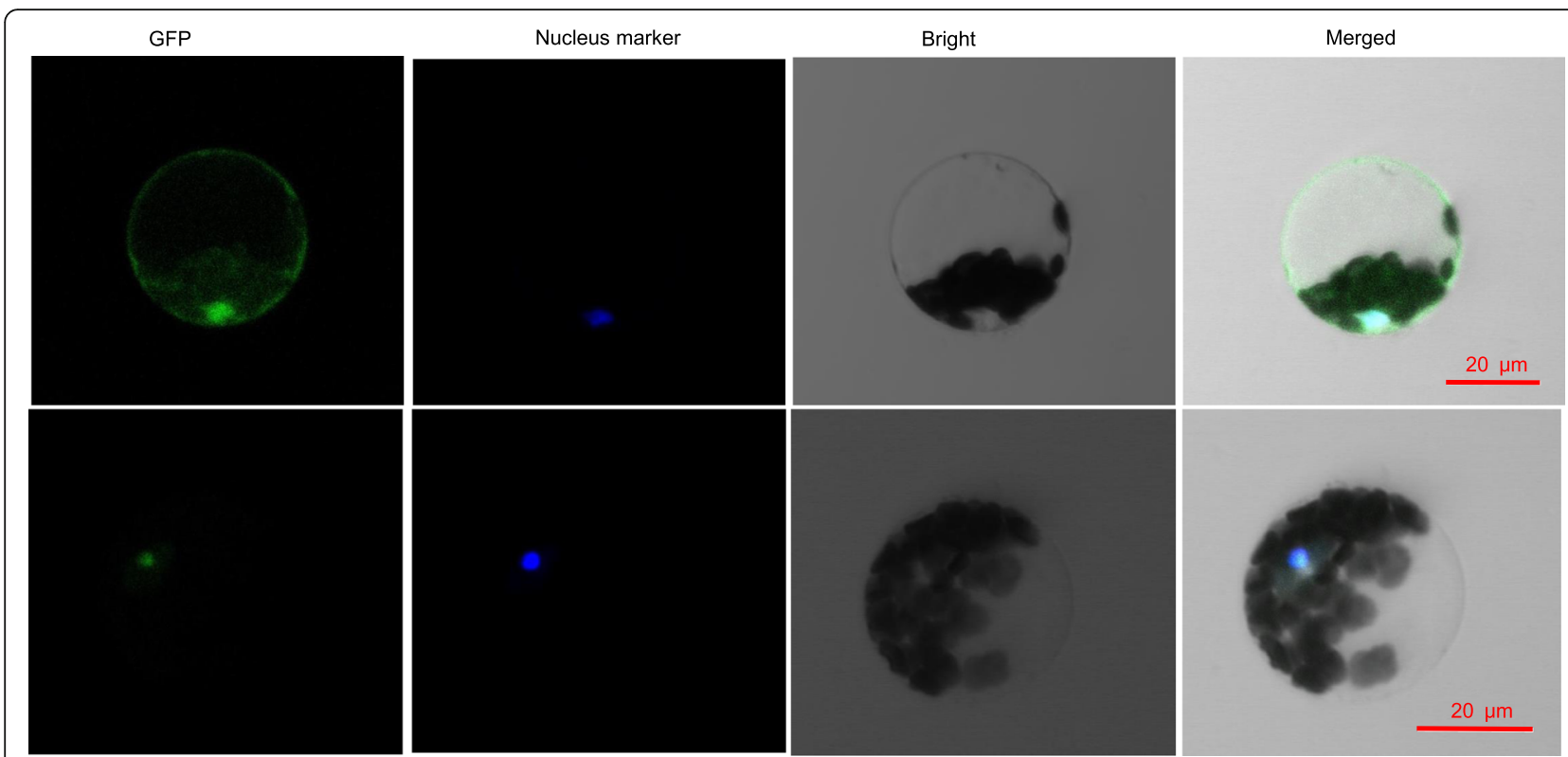

Fig. 4 Subcellular localization of GhREV2 in Arabidopsis protoplasts. GhREV2 localizes in nucleus. GFP or GhREV2-GFP was expressed in Arabidopsis protoplast. Protoplasts were isolated from leaves of 10-day-old Arabidopsis to express 355::GFP (top) or 355::GhREV2-GFP (bottom). The subcellular localization were examined using a confocal microscope. Bright is bright field. Bright field and green fluorescence images were merged. Scale bars $=20 \mu \mathrm{m}$

activated the luciferase reporter. The activation activity of GhREV2 was similar to that of AtWRKY29 (Asai et al. 2002; Li et al. 2017) (Fig. 3b).

\section{Subcellular localization of GhREV2}

To determine the subcellular localization, GhREV2 was fused with the $\mathrm{C}$-terminus of green fluorescent protein (GFP) and transformed into Arabidopsis protoplasts. The empty GFP construct was driven by the cauliflower mosaic virus $35 \mathrm{~S}$ promoter and expressed in the cytoplasm, nucleus, and plasma membrane of the protoplasts. The fluorescence signals derived from the GhREV2-GFP construct were observed only in nucleus (Fig. 4).

\section{Silencing of GhREV2 causes developmental defect in cotton SAM}

To characterize the function of GhREV2, we silenced it in cotton seedlings via the Tobacco rattle virus (TRV)-based VIGS system. After the VIGS-GLA1 plants showed albino phenotype, the relative expression levels were assessed using qRT-PCR. The data showed that not only GhREV2 but also GhREV1, GhREV3 and GhREV4 were silenced compared with those in the control due to the high similarity of GhREV genes. The silencing efficiency of GhREVs all exceeded 55\% (Fig. 5a).
After two months after the plants were treated with the VIGS system, the VIGS-GhREV2 plants exhibited an abnormal SAM, but not the VIGS-Ctrl plants (Fig. $5 \mathrm{~b})$. For the possible mechanism of this abnormality, we determined the relative expression level of WUSCHEL (GhWUSA10) and SHOOT MERISTEMLESS (GhSTM), two key genes involved in SAM development, found that the transcripts of both GhWUSA10 and GhSTM decreased by $50 \%$ in VIGSGhREV2 plants (Fig. 5c).

\section{Discussion}

Similar to AtREVs in Arabidopsis, GhREVs are expressed in various tissues in cotton (Fig. 2). We speculate that GhREVs may also be involved in the formation of vascular bundles (Ramachandran et al. 2016), in the establishment of leaf polarity (Kim et al. 2010; Xie et al. 2014), and in the differentiation of the SAM (Lee and Clark 2015; Mandel et al. 2016). The expression of GhREV2 and GhREV4 in the SAM was significantly higher than GhREV1 and GhREV3 (Fig. 2), indicating that GhREV2 and GhREV4 may act mainly in the development of the shoot apex.

In addition, double luciferase reporter assays showed that only GhREV2 and GhREV3 possess transcriptional activity (Fig. 3). Based on the spatio-temporal expression patterns, GhREV3 may acts as a positive TF in the stem to regulate the development of vascular tissues, while GhREV2 may play a major role in regulating the SAM. 


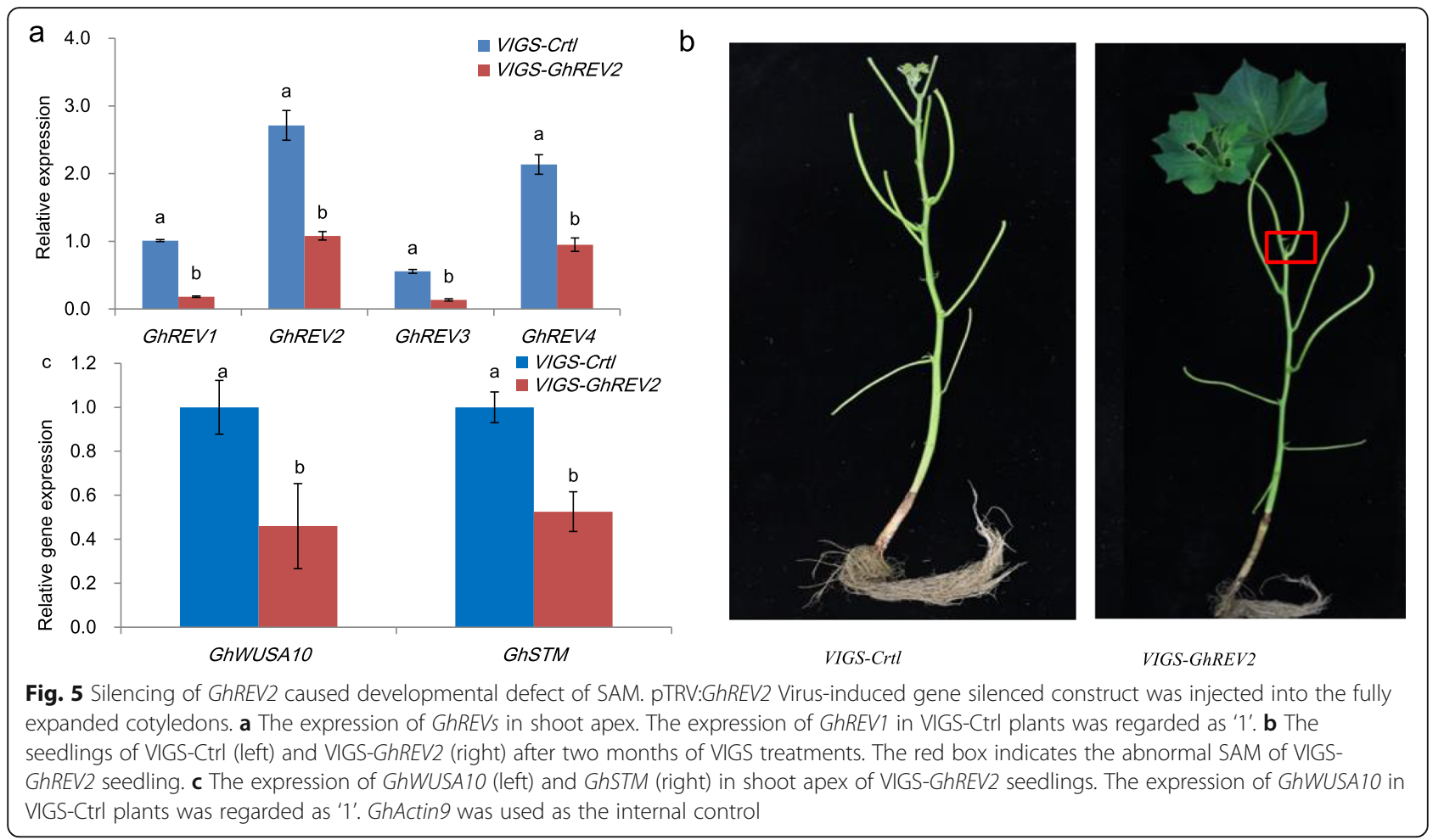

Although GhREV4 showed higher expression levels than GhREV1 and GhREV3 in all tested tissues, it does not function as a transcriptional activator. Moreover, GhREV2 was located in the nucleus (Fig. 4), as expected with its TF function.

Owing to the high homology of GhREVs, the silencing of GhREV2 also reduced the expression level of other family members to a certain extent. However, the transcriptional activity assay showed that only GhREV2 and GhREV3 had transcriptional activity, and the expression of GhREV3 was less in the SAM. Therefore, we speculated that GhREV2 plays the major function in controlling the development of the SAM.

Extensive molecular genetic studies have identified key regulators and networks that operate in the SAM processes across species. It is known that the homeodomain of WUSCHEL (WUS) TF is essential for the maintenance of stem cells in plant SAM. WUS expresses in the OC (Mayer et al. 1998), and then enters the CZ and activates the transcription of CLAVATA3 (CLV3) (Yadaw et al. 2012; Daum et al. 2014). In turn, CLV3 can repress WUS expression. These events form a negative feedback loop that guarantees the dynamic size adjustment of the stem cell niches in the SAM (Clark 1997; Schoof et al. 2000; Lenhard and Laux 2003; Gaillochet and Lohmann 2015). In addition, the SHOOTMERISTEMLESS (STM) is a member of the KNOX family and it prevents stem cell differentiation by inhibiting the expression of organforming factors ASYMMETRIC LEAVES1 (AS1) and AS2 in the CZ (Katayama et al. 2010). The mutation of STM can lead to premature termination of the stem and meristem. That is in parallel with WUS-CLV3 pathway (Clark et al. 1996; Endrizzi et al. 2010). Importantly, it has been reported that HD-ZIP III family, including REV and PHB, can strongly interact with B-type ARABIDOPSIS RESPONSE REGULATORs (ARRs) to activate WUS (Zhang et al. 2017). In this study, we found that the expression of GhWUSA10 and GhSTM is explicitly suppressed in VIGS-GhREV2 plants, indicating that GhREV2 may function together with GhWUSA10 and GhSTM to regulate the development of cotton SAM.

\section{Conclusion}

The results in this study indicate that GhREV2, a nuclear localized transcriptional activator, positively affects the development of cotton SAM, potentially by modulating the transcripts of GhWUSA10 and GhSTM.

\section{Supplementary information}

Supplementary information accompanies this paper at https://doi.org/10. 1186/s42397-020-0045-y.

Additional file 1: Table S1. Primer sequences. 


\section{Acknowledgments}

We appreciate Prof. Shan, Libo of Texas A\&M University for providing the VIGS constructs. We thank Dr. Cox, Kevin L. Jr. for critical reading of the manuscript. The authors have declared no conflict of interests.

\section{Author details}

College of Agronomy and Biotechnology, China Agricultural University, Beijing 100 193, China.

\section{Authors' contributions}

Tian XL and Li ZH conceived and designed the study; An J and Yang DD carried out the experiments, analyzed and interpreted the data; Yang DD, Li FJ and Tian XL prepared the manuscript. Eneji AA revised the manuscript. All authors read and approved the final version of manuscript.

\section{Funding}

This work was supported by The National Natural Science Foundation of China (31571588).

\section{Availability of data and materials}

No other data related to this study is available at this time.

\section{Ethics approval and consent to participate}

Not applicable.

\section{Consent for publication}

Not applicable.

\section{Competing interests}

The authors declare that they have no competing interests.

\section{Author details}

'Department of Crop Physiology and Cultivation, College of Agronomy and Biotechnology, China Agricultural University, Beijing 100193, China. ${ }^{2}$ Department of Soil Science, Faculty of Agriculture, Forestry and Wildlife Resources Management, University of Calabar, Calabar, Nigeria.

\section{Received: 24 September 2019 Accepted: 12 January 2020}

\section{Published online: 11 February 2020}

\section{References}

Asai T, Tena G, Plotnikova J, et al. MAP kinase signalling cascade in Arabidopsis innate immunity. Nature. 2002:415(6875):977. https://doi.org/10.1038/ 415977a.

Baima S, Nobili F, Sessa G, et al. The expression of the ATHB-8 homeobox gene is restricted to provascular cells in Arabidopsis thaliana. Development. 1995: 121(12):4171-82. https://doi.org/10.1111/j.1365-2303.1995.tb00491.x.

Bustamante M, Matus JT, Riechmann JL. Genome-wide analyses for dissecting gene regulatory networks in the shoot apical meristem. J Exp Bot. 2016;67(6): 1639-49. https://doi.org/10.1093/jxb/erw058.

Byrne ME. Shoot meristem function and leaf polarity: the role of class III HD-ZIP genes. PLoS Genet. 2006;2(6):e89. https://doi.org/10.1371/journal.pgen. 0020089

Gaillochet C, Lohmann JU. The never-ending story: from pluripotency to plant developmental plasticity. Development. 2015;142(13):2237-49. https://doi. org/10.1242/dev.117614

Clark SE. Organ formation at the vegetative shoot meristem. Plant Cell. 1997:9(7): 1067-76. https://doi.org/10.1105/tpc.9.7.1067.

Clark SE, Jacobsen SE, Levin J Z, et al. The CLAVATA and SHOOT MERISTEMLESS loci competitively regulate meristem activity in Arabidopsis.Development. 1996; 122(5):1567-75.

Emery JF, Floyd SK, Alvarez J, et al. Radial patterning of Arabidopsis shoots by class III HD-ZIP and KANADI genes. Curr Biol. 2003;13(20):1768-74. https://doi. org/10.1016/j.cub.2003.09.035.

Endrizzi K, Moussian B, Haecker A, et al. The SHOOT MERISTEMLESS gene is required for maintenance of undifferentiated cells in Arabidopsis shoot and floral meristems and acts at a different regulatory level than the meristem genes WUSCHEL and ZWILLE. Plant J. 2010;10(6):967-79. https://doi.org/10. 1046/j.1365-313x.1996.10060967.x.
Daum G, Medzihradszky A, Suzaki T, et al. A mechanistic framework for noncell autonomous stem cell induction in Arabidopsis. Proc Natl Acad Sci U S A. 2014;111(40):14619-24. https://doi.org/10.1073/pnas.1406446111.

Green KA, Prigge MJ, Katzman RB, et al. CORONA, a member of the class III homeodomain leucine zipper gene family in Arabidopsis, regulates stem cell specification and organogenesis. Plant Cell. 2005;17(3):691-704. https://doi. org/10.1105/tpc.104.026179

Bäurle I, Laux T. Apical meristems: the plant's fountain of youth. Bioessays. 2003; 25(10):961-70. https://doi.org/10.1002/bies.10341.

Kim HS, Kim SJ, Abbasi N, et al. The DOF transcription factor Dof5. 1 influences leaf axial patterning by promoting Revoluta transcription in Arabidopsis. Plant J. 2010;64(3):524-35. https://doi.org/10.1111/j.1365-313X.2010.04346.X.

Mukherjee K, Bürglin TR. MEKHLA, a novel domain with similarity to PAS domains, is fused to plant homeodomain-leucine zipper III proteins. Plant Physiol. 2006;140(4):1142-50. https://doi.org/10.1104/pp.105.073833.

Lee C, Clark SE. A WUSCHEL-independent stem cell specification pathway is repressed by PHB, PHV and CNA in Arabidopsis. PLoS One. 2015;10(5): e0126006. https://doi.org/10.1371/journal.pone.0126006.

Li F, Fan G, Lu C, et al. Genome sequence of cultivated upland cotton (Gossypium hirsutum TM-1) provides insights into genome evolution. Nat Biotechnol. 2015:33(5):524-30. https://doi.org/10.1038/nbt.3208.

Li F, Li M, Wang P, et al. Regulation of cotton (Gossypium hirsutum) drought responses by mitogen-activated protein (MAP) kinase cascade-mediated phosphorylation of GhWRKY59. New Phytol. 2017;215(4):1462-75. https://doi. org/10.1111/nph. 14680

Mandel T, Candela H, Landau U, et al. Differential regulation of meristem size, morphology and organization by the ERECTA, CLAVATA and class III HD-ZIP pathways. Development. 2016;143(9):1612-22. https://doi.org/10.1242/dev.129973.

Mayer KF, Schoof H, Haecker A, et al. Role of WUSCHEL in regulating stem cell fate in the Arabidopsis shoot meristem. Cell. 1998;95(6):805. https://doi.org/10. 1016/s0092-8674(00)81703-1.

McConnell JR, Emery J, Eshed Y, et al. Role of PHABULOSA and PHAVOLUTA in determining radial patterning in shoots. Nature. 2001;411(6838):709-13. https:/doi. org/10.1038/35079635.

Lenhard M, Laux T. Stem cell homeostasis in the Arabidopsis shoot meristem is regulated by intercellular movement of CLAVATA3 and its sequestration by CLAVATA1. Development. 2003;130(14):3163-73. https:/doi.org/10.1242/dev.00525.

Pautler M, Tanaka W, Hirano HY, et al. Grass meristems l: shoot apical meristem maintenance, axillary meristem determinacy and the floral transition. Plant Cell Physiol. 2013;54(3):302-12. https:/doi.org/10.1093/pcp/pct025.

Mu C, Zhou L, Shan L, et al. Phosphatase GhDs PTP 3a interacts with annexin protein Gh ANN 8b to reversely regulate salt tolerance in cotton (Gossypium spp.). New Phytol. 2019. https://doi.org/10.1111/nph.15850.

Katayama N, Koi S, Kato M. Expression of SHOOT MERISTEMLESS, WUSCHEL, and ASYMMETRIC LEAVES1 homologs in the shoots of Podostemaceae: implications for the evolution of novel shoot organogenesis. Plant Cell. 2010; 22(7):2131-40. https://doi.org/10.1105/tpc.109.073189.

Otsuga D, DeGuzman B, Prigge MU, et al. REVOLUTA regulates meristem initiation at lateral positions. Plant J. 2001;25(2):223-36. https://doi.org/10.1046/.1365-313x.2001.00959x.

Ponting CP, Aravind L. START: a lipid-binding domain in StAR, HD-ZIP and signalling proteins. Trends Biochem Sci. 1999:24(4):130-2. https//doi.org/10.1016/509680004(99)01362-6.

Prigge MJ, Denichiro O, Alonso JM, et al. Class III homeodomain-leucine zipper gene family members have overlapping, antagonistic, and distinct roles in Arabidopsis development. Plant Cell. 2005;17(1):61-76. https://doi.org/10. 1105/tpc.104.026161.

Du Q, Wang HZ. The role of HD-ZIP III transcription factors and miR165/166 in vascular development and secondary cell wall formation. Plant Signal Behav. 2015;10(10): e1078955. https:/doi.org/10.1080/15592324.2015.1078955.

Yadav RK, Perales M, Gruel J, et al. WUSCHEL protein movement mediates stem cell homeostasis in the Arabidopsis shoot apex. Genes Dev. 2012;25(5):202530. https://doi.org/10.1101/gad.17258511.

Ramachandran P, Carlsbecker A, Etchells JP. Class III HD-ZIPs govern vascular cell fate: an HD view on patterning and differentiation. J Exp Bot. 2016;68(1):55-69. https://doi. org/10.1093/jxb/erw370.

Schoof H, Lenhard M, Haecker A, et al. The stem cell population of Arabidopsis shoot meristems is maintained by a regulatory loop between the CLAVATA and WUSCHEL genes. Cell. 2000;100(6):635-44. https:/doi.org/10.1016/s0092-8674(00)80700-x.

Shi B, Zhang C, Tian C, et al. Two-step regulation of a meristematic cell population acting in shoot branching in Arabidopsis. PLoS Genet. 2016;12(7):e1006168. https:/doi.org/ 10.1371/journal.pgen.1006168. 
Soyars CL, James SR, Nimchuk ZL. Ready, aim, shoot: stem cell regulation of the shoot apical meristem. Curr Opin Plant Biol. 2016;29:163-8. https://doi.org/10. 1016/j.pbi.2015.12.002.

Talbert PB, Adler HT, Parks DW, et al. The REVOLUTA gene is necessary for apical meristem development and for limiting cell divisions in the leaves and stems of Arabidopsis thaliana. Development. 1995; 121(9):2723-35.

Turchi L, Baima S, Morelli G, et al. Interplay of HD-zip II and III transcription factors in auxin-regulated plant development. J Exp Bot. 2015;66(16):5043-53. https://doi.org/10.1093/jxb/erv174.

Xie Y, Huhn K, Brandt R, et al. REVOLUTA and WRKY53 connect early and late leaf development in Arabidopsis. Development. 2014;141(24):4772-83. https://doi. org/10.1242/dev.117689.

Kim YS, Kim SG, Lee M, et al. HD-ZIP III activity is modulated by competitive inhibitors via a feedback loop in Arabidopsis shoot apical meristem development. Plant Cell. 2008;20(4):920-33. https://doi.org/10.1105/tpc.107. 057448 .

Zhang TQ, Lian $\mathrm{H}$, Zhou CM, et al. A two-step model for de novo activation of WUSCHEL during plant shoot regeneration. Plant Cell. 2017;29(5):1073-87. https://doi.org/10.1105/tpc.16.00863

Ready to submit your research? Choose BMC and benefit from:

- fast, convenient online submission

- thorough peer review by experienced researchers in your field

- rapid publication on acceptance

- support for research data, including large and complex data types

- gold Open Access which fosters wider collaboration and increased citations

- maximum visibility for your research: over $100 \mathrm{M}$ website views per year

At $\mathrm{BMC}$, research is always in progress.

Learn more biomedcentral.com/submissions 